\title{
The Ontario Universal Typing of Tuberculosis (OUT-TB) Surveillance Program - what it means to you
}

\author{
Shelly Bolotin $\mathrm{PhD}^{1}$, David C Alexander $\mathrm{PhD}^{1}$, Jennifer L Guthrie MSc${ }^{1}$, \\ Steven J Drews $\mathrm{PhD}^{1,2}$, Frances Jamieson $\mathrm{MD}^{1,2}$
}

S Bolotin, DC Alexander, JL Guthrie, SJ Drews, F Jamieson. The Ontario Universal Typing of Tuberculosis (OUT-TB) Surveillance Program - what it means to you. Can Respir J 2010;17(3):e51-e54.

BACKGROUND: Tuberculosis (TB) is a serious disease that is transmitted primarily by the airborne route. Effective disease control and outbreak management requires the timely diagnosis, isolation and treatment of infected individuals with active disease; contact tracing to identify secondary cases likely to benefit from treatment of latent infection; and laboratory identification or confirmation of epidemiologically linked cases. TB genotyping enables the comparison of Mycobacterium tuberculosis complex (MTBC) strains and the identification of cases that may or may not be linked. The increased availability of molecular methods for genotyping has allowed for greater discrimination of MTBC strains and greatly enhanced understanding of $\mathrm{TB}$ transmission patterns.

OBJECTIVE: To improve TB surveillance and control in Ontario, the Public Health Laboratories of the Ontario Agency for Health Protection and Promotion has introduced the Ontario Universal Typing of Tuberculosis (OUT-TB) Surveillance Program.

METHODS: The first isolate from every new TB case will be genotyped with two rapid molecular methods: spoligotyping and mycobacterial interspersed repetitive unit-variable-number tandem repeat typing. MTBC isolates with nonunique genotypes and, thus, potentially linked to other TB cases, will also be genotyped by IS6110 restriction fragment length polymorphism analysis.

CONCLUSION: By providing TB control programs using these new genotyping tools, and using traditional and new case investigation methods (eg, social network analysis), this new program will provide a clearer picture of TB in Ontario, and permit more effective use of public health resources and improve disease control.

Key Words: Genotyping; Public health; Tuberculosis

\section{WHAT IS THE 'ONTARIO \\ UNIVERSAL TYPING OF TUBERCULOSIS SURVEILLANCE PROGRAM'?}

$\mathrm{T}_{\mathrm{s}}^{\mathrm{h}} \mathrm{orm}$ he Ontario Universal Typing of Tuberculosis (OUT-TB)

Surveillance Program aims to improve surveillance and control of tuberculosis (TB) disease in Ontario by characterizing Mycobacterium tuberculosis complex (MTBC) isolates from every new case of TB in Ontario with a set of molecular genotyping tools. The present review explains the rationale behind the implementation of this program in Ontario and provides guidelines for interpretation of MTBC genotypes using the new methodologies.

Why should there be 'universal surveillance' of TB? Until recently, genotyping of MTBC isolates was performed by
Le typage universel du programme de surveillance de la tuberculose en Ontario - ce qu'il signifie pour vous

HISTORIQUE : La tuberculose (TB) est une maladie grave qui est surtout transmise par voie aéroportée. Le contrôle efficace des maladies et la prise en charge des flambées exigent un diagnostic rapide, l'isolement et le traitement des personnes infectées par une maladie active, le dépistage des contacts pour repérer les cas secondaires susceptibles de profiter du traitement d'une infection latente et le dépistage ou la confirmation en laboratoire de cas liés sur le plan épidémiologique. Le génotypage de la TB permet de comparer les souches complexes de la Mycobacterium tuberculosis (CMTB) et de déceler les cas qui peuvent ou non y être liés. Puisque les méthodes moléculaires de génotypage sont plus accessibles, il est possible de mieux discriminer les souches de CMTB et de beaucoup mieux comprendre les profils de transmission de la TB.

OBJECTIF : Pour améliorer la surveillance et le contrôle de la TB en Ontario, les Laboratoires de santé publique de l'Agence ontarienne de protection et de promotion de la santé ont lancé le typage universel du programme de génotypage de la tuberculose de l'Ontario.

MÉTHODOLOGIE : Le premier isolat de chaque nouveau cas de TB sera génotypé au moyen de deux méthodes moléculaires rapides : le spoligotypage et le typage des unités mycobactériennes disséminées répétées et des répétitions en tandem en nombre variable. Les isolats de CMTB dont les génotypes ne sont pas uniques et qui sont donc potentiellement liés aux autres cas de TB seront également génotypés par une analyse de la séquence d'insertion 6110 du polymorphisme de restriction.

CONCLUSION : En offrant des programmes de contrôle de la TB au moyen de ces nouveaux outils de génotypage et à l'aide de méthodes d'exploration classiques et nouvelles (p. ex., analyse des réseaux sociaux), ce nouveau programme donnera un portrait plus clair de la TB en Ontario, sans compter qu'il assurera une utilisation plus efficace des ressources de santé publique et un meilleur contrôle de la maladie.

the Ontario Public Health Laboratories (PHL) only on request. Genotyping all new cases of TB will aid contact investigations because the source of a TB infection is not always obvious, and exposure may be a result of 'social networking' (eg, common point of interaction with infectious individuals) or high-risk behaviours $(1,2)$ that are not always uncovered during patient interviews. Switching from the 'request only' to a 'universal surveillance' approach means that links between apparently unrelated cases will be identified. Although it was traditionally believed that only $10 \%$ of TB infections were due to recent transmission, the implementation of universal genotyping surveillance programs in many jurisdictions has demonstrated that this is not the case. Studies in cities such as New York and San Francisco (USA) have shown that new infections can comprise as many as $40 \%$ of all cases $(3,4)$.

${ }^{1}$ Public Health Laboratory - Toronto, Ontario Agency for Health Protection and Promotion; ${ }^{2}$ Department of Laboratory Medicine and

Pathobiology, University of Toronto, Toronto, Ontario

Correspondence and reprints: Dr Frances Jamieson, TB and Mycobacteriology, Public Health Laboratories - Toronto, Ontario Agency for Health

Protection and Promotion, 81 Resources Road, Toronto, Ontario M9P 3T1. Telephone 416-235-5841, fax 416-235-5951,

e-mail frances.jamieson@oahpp.ca 


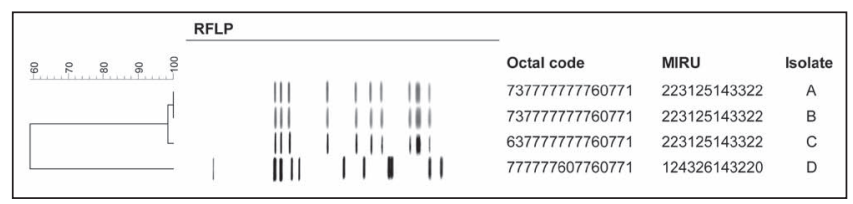

Figure 1) Dendrogram demonstrating the genotyping analysis of four patient isolates using restriction fragment length polymorphisms (RFLP), spoligotyping and mycobacterial interspersed repetitive unit-variable-number tandem repeat (MIRU-VNTR) analysis. Isolates $\mathrm{A}$ and $\mathrm{B}$ are identical by all methods and are considered to be identical strains. Isolate $\mathrm{C}$ is a closely related strain. It shares MIRU-VNTR and RFLP patterns with isolates A and B, but demonstrates a single-digit difference by spoligotyping. Isolate $D$ is distinct by all three genotyping methods and, thus, is considered to be unrelated

In addition to revealing previously unknown transmission networks, universal genotyping ('fingerprinting') can be used to monitor and manage TB within a population. For example, it may help to identify populations at higher risk for TB transmission. This was the outcome of a genotyping study in San Francisco (5), which demonstrated that homeless patients who were also HIV positive were found to have a clustering rate of $88 \%$, indicating that this population is at high risk for $\mathrm{TB}$ transmission.

Another important facet of universal TB genotyping is the ability to differentiate between reactivation of latent TB and recent transmission $(2,6-8)$. This can prevent potentially expensive and prolonged contact investigations, and may be especially important when examining TB transmission patterns in certain immigrant populations from countries with a high burden of TB. For example, a study performed in Montreal (Quebec) (9) found that MTBC isolates from immigrants of Haitian origin had a high frequency of matching genotypes. This could suggest either recent transmission within the Haitian community or a limited diversity of MTBC strains acquired in Haiti. To determine whether the matching genotypes were due to a recent outbreak within the community, the genotypes of isolates from Haitian patients were compared with isolates from non-Haitian, foreign-born TB patients. If the genotypes of TB strains from Haitian patients were similar because they originated from a common ancestor strain, these genotypes should be more similar to each other than to those obtained from a diverse group of other foreign-born patients. After calculating genetic distance - a method that quantifies the relationship between isolates - it was found that the Haitian and non-Haitian isolates shared similar genetic distances, indicating that the increased clustering seen in the Haitian community is probably a result of disease transmission on arrival in Canada and not due to a limited number of endemic strains acquired in Haiti (9-11).

Although TB genotyping is used most often for contact investigations and outbreak management, the availability of TB fingerprints also enables public health authorities to monitor the circulation of drug-resistant strains. Although the genetic mutations that confer drug resistance do not usually change the fingerprint of an isolate, genetic analysis may allow 'flagging' of TB cases that share DNA fingerprints with isolates that are drug resistant. It may also shed light on the evolution of drug resistance in a particular strain. In addition, universal
TB genotyping has been found to be useful in other jurisdictions where several laboratories provide TB diagnostic services to identify false-positive results due to laboratory contamination, which is believed to occur in up to $3 \%$ of cases in the United States (12).

\section{Why should multiple molecular genotyping methods be used?}

Historically, the PHL has used IS6110 restriction fragment length polymorphism (RFLP) analysis, the gold-standard method for MTBC strain analysis (13). Although usually satisfactory for linking patients who are direct contacts, some MTBC strains are not effectively typed by RFLP (14). Moreover, genotyping by any single method may incorrectly link patients with similar but nonidentical strains.

The enhanced genetic analysis and improved interpretation of strain relatedness provided by the use of multiple molecular genotyping methods in the OUT-TB surveillance program will better serve the public health units conducting the contact tracing and outbreak analysis. The strength of molecular surveillance has been repeatedly demonstrated in the literature. Several molecular epidemiology studies $(3,4,15)$ have shown that using a systematic, population-based typing approach is the only way to identify all transmission networks and disease clusters.

\section{How will the program be implemented?}

Universal genotyping of MTBC strains will enhance the PHL $\mathrm{TB}$ and mycobacteriology diagnostic and reference laboratory services for public health stakeholders and the health care community. In Ontario, the PHL processes almost all specimens submitted for diagnostic testing for TB. TB case isolates are also submitted to the PHL for confirmation and susceptibility testing from private and hospital laboratories that provide MTBC culture services. This ensures that there is a standardized approach to the identification, characterization and detection of drug-resistant isolates, as well as genotyping.

Universal genotyping will be performed using three methods: spoligotyping, mycobacterial interspersed repetitive unitvariable-number tandem repeat (MIRU-VNTR) analysis, and IS6110 RFLP.

Spoligotyping is a rapid genotyping method that combines nucleic acid amplification (eg, polymerase chain reaction [PCR]) technology with DNA hybridization to detect variability in the direct repeat (DR) region of the MTBC genome. Each isolate is tested for the presence or absence of 43 short DR sequences (16). If a specific spacer is present in the DR region, it will be amplified by PCR and, when hybridized to the spoligotyping membrane, will appear as a black spot. If the spacer is absent from the DR region, no amplification or hybridization will occur and the corresponding space on the membrane will be blank. Finally, the pattern of 43 'spots' is translated into a 15-digit number called the 'octal code' (Figure 1).

MIRU-VNTR analysis is also a rapid genotyping method (17). Similar to spoligotyping, it is PCR-based and targets nucleotide repeat sequences found in the MTBC genome. Unlike spoligotyping, it does not target the DR region, but a set of distinct 'MIRU-VNTR' loci. At each locus, the number of repeat units (which may vary between strains) are determined by using a fragment analysis protocol that measures the size of the PCR product obtained at each locus $(18,19)$. 
TABLE 1

Interpretation of laboratory genotyping results (comparison of two isolates)

\begin{tabular}{|c|c|c|}
\hline Laboratory method & Interpretation & Reason \\
\hline Strains identical by all methods & Strains are identical & All methods concur that isolates are genotypically identical \\
\hline $\begin{array}{l}\text { Spoligotyping and MIRU-VNTR types are identical, RFLP } \\
\text { patterns differ by one band }\end{array}$ & Strains closely related & $\begin{array}{l}\text { RFLP is more discriminatory than the two rapid typing methods. } \\
\text { Epidemiological data required }\end{array}$ \\
\hline $\begin{array}{l}\text { Spoligotyping and MIRU-VNTR types are identical, RFLP } \\
\text { patterns differ by more than one band }\end{array}$ & Strains unrelated & RFLP is more discriminatory than the two rapid typing methods \\
\hline $\begin{array}{l}\text { Spoligotyping or MIRU-VNTR differs by one digit, RFLP } \\
\text { patterns are identical }\end{array}$ & Strains closely related & $\begin{array}{l}\text { Strains are closely related, but mutation may have occurred. } \\
\text { Epidemiological data required }\end{array}$ \\
\hline $\begin{array}{l}\text { Spoligotyping or MIRU-VNTR types are different, RFLP } \\
\text { patterns differ by more than one band }\end{array}$ & Strains unrelated & All methods indicated strains are not related \\
\hline
\end{tabular}

MIRU-VNTR Mycobacterial interspersed repetitive unit-variable-number tandem repeat; RFLP Restriction fragment length polymorphism

Standardized nomenclature is used to create a digital code representing the variable number of repeats at each locus (Figure 1). The original method targeted 12 loci (thus generating a 12-digit code); however, more recently, higher resolution strategies have been promoted $(20,21)$. A 24-loci MIRUVNTR method that improves strain differentiation and reduces 'pseudoclustering' of unrelated isolates has been implemented at the PHL $(22,23)$.

The MTBC-specific mobile genetic element IS6110 can replicate and insert itself into the $\mathrm{TB}$ genome many times; RFLP genotyping exploits this phenomenon. The number and position of bands in an RFLP profile corresponds to the number and position of IS6110 elements, respectively, in a strain's genome. Strains that are identical or closely related will have identical or nearly identical band patterns, while unrelated strains will have different patterns (Figure 1). RFLP produces the highest resolution between strains (24), is considered the gold standard and previously was the most widely used genotyping method for MTBC. However, it is very labour intensive, requires large quantities of DNA (dependent on organism growth, which requires several weeks) and cannot be reliably used to type strains with fewer than six IS6110 insertions (ie, less than six bands in the RFLP pattern). Furthermore, there are technical difficulties with interlaboratory variation (25), limiting its usefulness as a singular typing method (14).

Through the OUT-TB program, the first isolate from every new TB case will be analyzed by spoligotyping and MIRUVNTR analysis. If initial genotyping results reveal that an isolate matches another strain in the OUT-TB database, RFLP analysis will be performed to determine whether the isolates are identical, related or unrelated. Genotypically identical isolates are of special interest to TB control efforts because they are indicative of recent transmission or a TB outbreak. Results from the rapid methods will be reported first. If RFLP is performed, those results will be provided as they become available. The implementation and subsequent interpretation of data from multiple methods overcomes the challenges and limitations associated with each individual genotyping method. As such, OUT-TB aims to provide the most comprehensive analysis possible and, therefore, the most useful data for TB control program personnel. However, universal genotyping cannot replace traditional TB control efforts. Appropriate interpretation of these data and confirmation of strain relatedness or potential transmission requires that public health units consider genotyping results in conjunction with the case investigation information.
How should the genotyping results be interpreted?

Generally, isolates that share identical DNA fingerprints are considered to be 'clustered' (ie, belonging to one group) and are believed to be related, possibly as a result of direct TB transmission. Isolates that are genotypically distinct from one another are considered to be unrelated $(3,4)$.

Isolates that are identical by MIRU-VNTR and spoligotyping analysis will then be analyzed by RFLP. If the RFLP patterns also match, the isolates will be considered to be clustered and identical. Because RFLP is not a rapid method and results may not be immediately available, cases that are considered to be identical by the two rapid methods should be further investigated by the TB control program. Once full genotyping is complete, isolates that match by only two out of the three typing methods will not be considered identical, with the final report reflecting the degree of relatedness (Table 1). For example, if isolates match by two methods and the third technique reveals a similar - but not identical - pattern, they may be reported as 'closely related'. Closely related strains may differ by one RFLP band (ie, an extra band, a missing band or a shifted band), one spoligotyping digit or one MIRU-VNTR digit (Figure 1). Epidemiological data must then be used to decide whether this strain relatedness represents recent $\mathrm{TB}$ transmission. It is important to note that, until the OUT-TB genotyping database is sufficiently populated, it is possible that some transmission events may not be detected.

Occasionally, two patients can have isolates with identical DNA fingerprints but no clear epidemiological links (Table 2). Several scenarios may account for this including laboratory error, sample contamination or, in communities where $\mathrm{TB}$ is endemic, the presence of a strain with a common genotype. To rule out these possibilities, the laboratory will investigate all identical isolates, particularly those that are temporally related (eg, from samples received on the same day or genotyped at the same time). In cases for which no confounding factors are identified, in-depth investigation by TB control personnel is necessary to uncover connections and risk factors that may not have been apparent during the initial investigation.

Although DNA fingerprints can be stable for long periods (eg, multiple rounds of bacterial division or several transmission cycles), mutations can occur, resulting in nonidentical strains despite an epidemiological link (Table 2). Rarely, 'mixed infections' by two different strains in a patient have been reported. When processed in the laboratory, a sample from a patient with a mixed infection may only grow strain 'A', 


\section{TABLE 2}

\section{Possible interpretations of discordant laboratory and} epidemiological results

\begin{tabular}{ll}
$\begin{array}{l}\text { Matching genotypes, } \\
\text { no epidemiological link }\end{array}$ & $\begin{array}{l}\text { Nonmatching genotypes, } \\
\text { direct epidemiological link }\end{array}$ \\
\hline $\begin{array}{l}\text { Endemic strain } \\
\text { Poorly differentiated by current } \\
\text { molecular typing methods }\end{array}$ & $\begin{array}{l}\text { Evolutionary drift } \\
\text { Mixed infection }\end{array}$ \\
Laboratory error & Laboratory error \\
Unknown epidemiological link & False epidemiological link \\
\hline
\end{tabular}

even though the patient transmits strain ' $\mathrm{B}$ ' to a contact. In this case, the two patients will appear to have nonidentical isolates even though direct transmission has occurred and an epidemiological link exists (25). Therefore, epidemiological and genotyping data together must drive the cluster analysis, requiring strong communication between the laboratory and TB control programs in the community. As OUT-TB accumulates both types of data, a clearer picture of TB transmission and disease in Ontario will become apparent.

\section{REFERENCES}

1. Murray M. Sampling bias in the molecular epidemiology of tuberculosis. Emerg Infect Dis 2002;8:363-9.

2. Miller AC, Sharnprapai S, Suruki R, et al. Impact of genotyping of Mycobacterium tuberculosis on public health practice in Massachusetts. Emerg Infect Dis 2002;8:1285-9.

3. Alland D, Kalkut GE, Moss AR, et al. Transmission of tuberculosis in New York City. An analysis by DNA fingerprinting and conventional epidemiologic methods. N Engl J Med 1994;330:1710-6.

4. Small PM, Hopewell PC, Singh SP, et al. The epidemiology of tuberculosis in San Francisco. A population-based study using conventional and molecular methods. N Engl J Med 199416;330:1703-9.

5. Moss AR, Hahn JA, Tulsky JP, Daley CL, Small PM, Hopewell PC. Tuberculosis in the homeless. A prospective study. Am J Respir Crit Care Med 2000;162(2 Pt 1):460-4.

6. Bandera A, Gori A, Catozzi L, et al. Molecular epidemiology study of exogenous reinfection in an area with a low incidence of tuberculosis. J Clin Microbiol 2001;39:2213-8.

7. Jasmer RM, Bozeman L, Schwartzman K, et al. Recurrent tuberculosis in the United States and Canada: Relapse or reinfection? Am J Respir Crit Care Med 2004;170:1360-6.

8. van RA, Warren R, Richardson M, et al. Exogenous reinfection as a cause of recurrent tuberculosis after curative treatment. N Engl J Med 1999;341:1174-9.

9. Haase I, Olson S, Behr MA, et al. Use of geographic and genotyping tools to characterise tuberculosis transmission in Montreal. Int J Tuberc Lung Dis 2007;11:632-8.

10. Kulaga S, Behr M, Musana K, et al. Molecular epidemiology of tuberculosis in Montreal. CMAJ 2002;167:353-4.

11. Kulaga S, Behr M, Nguyen D, et al. Diversity of Mycobacterium tuberculosis isolates in an immigrant population: Evidence against a founder effect. Am J Epidemiol 2004;159:507-13.

12. Burman WJ, Reves RR. Review of false-positive cultures for Mycobacterium tuberculosis and recommendations for avoiding unnecessary treatment. Clin Infect Dis 2000;31:1390-5.

13. van Embden JD, Cave MD, Crawford JT, et al. Strain identification of Mycobacterium tuberculosis by DNA fingerprinting: Recommendations for a standardized methodology. J Clin Microbiol 1993;31:406-9.

14. Mathema B, Kurepina NE, Bifani PJ, Kreiswirth BN. Molecular epidemiology of tuberculosis: Current insights. Clin Microbiol Rev 2006;19:658-85.
Because the OUT-TB program is based on TB control approaches in other jurisdictions that may differ from approaches in Ontario, the cooperation of all stakeholders during program implementation will ensure that any shortcomings are identified in a timely manner. As the OUT-TB program is used, factors such as the number of recent infections versus remote infections, the diversity of strains and the number of clustered cases will be determined. This will provide an accurate snapshot of TB disease in Ontario, allowing for program optimization.

It is expected that the OUT-TB Surveillance Program will be a useful tool for TB control programs in the province, enabling programs to provide efficient and effective TB control services such that the spread of TB within the province, as well as outside Ontario's borders, is minimized as much as possible.

CONFLICT OF INTEREST: The authors declare no conflicts of interest. This project was partially funded by a grant from the Ideas and Innovations Fund, Ontario Ministry of Government and Consumer Services.
15. Gutierrez MC, Vincent V, Aubert D, et al. Molecular fingerprinting of Mycobacterium tuberculosis and risk factors for tuberculosis transmission in Paris, France, and surrounding area. J Clin Microbiol 1998;36:486-92.

16. Kamerbeek J, Schouls L, Kolk A, et al. Simultaneous detection and strain differentiation of Mycobacterium tuberculosis for diagnosis and epidemiology. J Clin Microbiol 1997;35:907-14.

17. Mazars E, Lesjean S, Banuls AL, et al. High-resolution minisatellite-based typing as a portable approach to global analysis of Mycobacterium tuberculosis molecular epidemiology. Proc Natl Acad Sci USA 2001;98:1901-6.

18. Supply P, Lesjean S, Savine E, Kremer K, van SD, Locht C. Automated high-throughput genotyping for study of global epidemiology of Mycobacterium tuberculosis based on mycobacterial interspersed repetitive units. J Clin Microbiol 2001;39:3563-71.

19. Oelemann MC, Diel R, Vatin V, et al. Assessment of an optimized mycobacterial interspersed repetitive unit-variable-number tandem-repeat typing system combined with spoligotyping for population-based molecular epidemiology studies of tuberculosis. J Clin Microbiol 2007;45:691-7.

20. Supply P, Allix C, Lesjean S, et al. Proposal for standardization of optimized mycobacterial interspersed repetitive unit-variablenumber tandem repeat typing of Mycobacterium tuberculosis. J Clin Microbiol 2006;44:4498-510.

21. Iwamoto T, Yoshida S, Suzuki K, et al. Hypervariable loci that enhance the discriminatory ability of newly proposed 15-loci and 24-loci variable-number tandem repeat typing method on Mycobacterium tuberculosis strains predominated by the Beijing family. FEMS Microbiol Lett 2007;270:67-74.

22. van Soolingen D, Hermans PW. Epidemiology of tuberculosis by DNA fingerprinting. Eur Respir J Suppl 1995;20:649s-56s.

23. Yeh RW, Hopewell PC, Daley CL. Simultaneous infection with two strains of Mycobacterium tuberculosis identified by restriction fragment length polymorphism analysis. Int J Tuberc Lung Dis 1999;3:537-9.

24. Kremer K, van SD, Frothingham R, et al. Comparison of methods based on different molecular epidemiological markers for typing of Mycobacterium tuberculosis complex strains: Interlaboratory study of discriminatory power and reproducibility. J Clin Microbiol 1999;37:2607-18.

25. Bauer J, Andersen AB, Kremer K, Miorner H. Usefulness of spoligotyping to discriminate IS6110 low-copy-number Mycobacterium tuberculosis complex strains cultured in Denmark. J Clin Microbiol 1999;37:2602-6. 


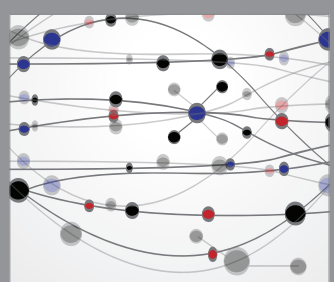

The Scientific World Journal
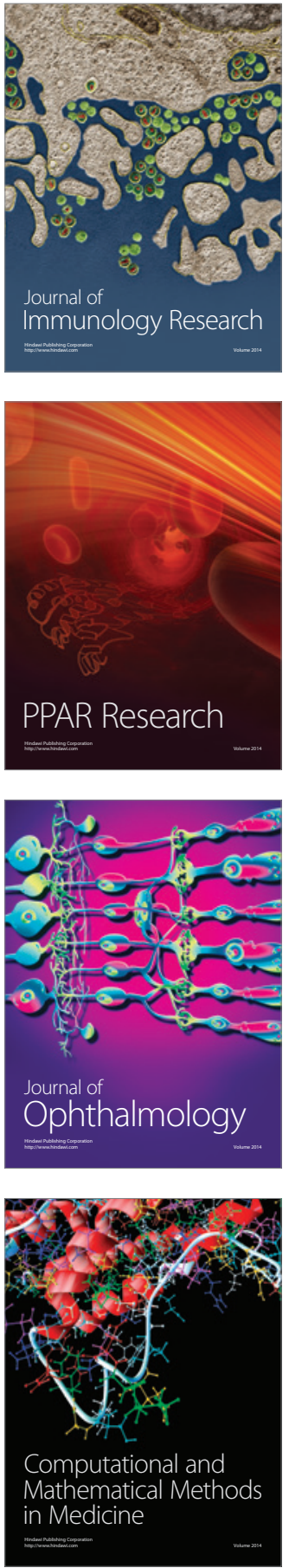

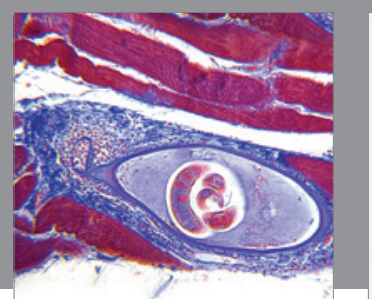

Gastroenterology Research and Practice

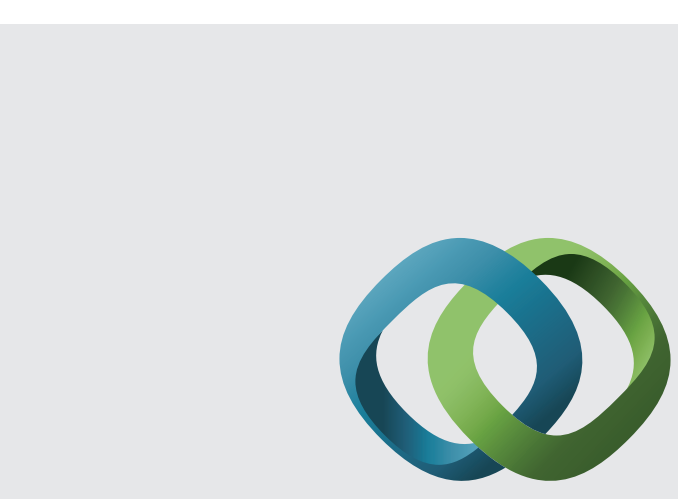

\section{Hindawi}

Submit your manuscripts at

http://www.hindawi.com
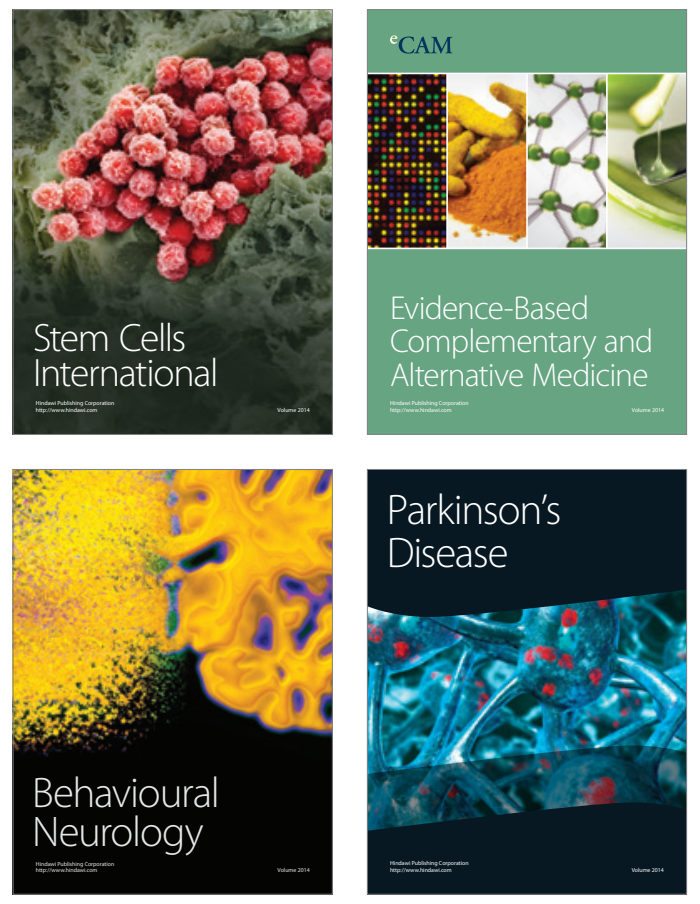
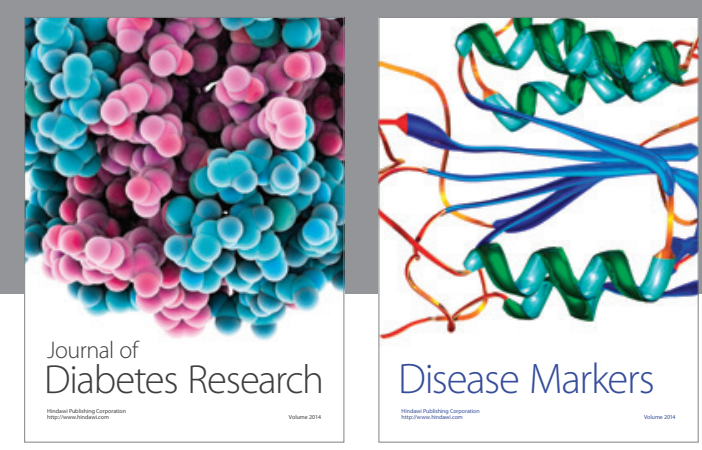

Disease Markers
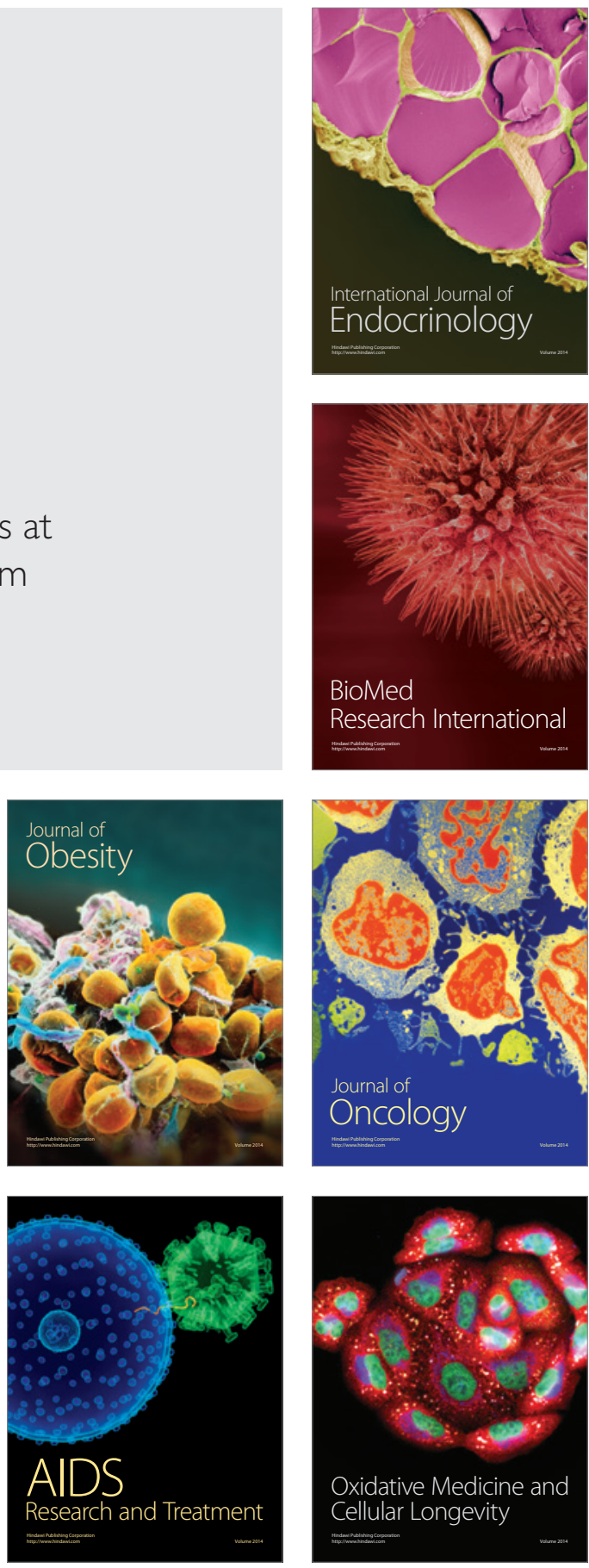